\title{
1 Interpretability of statistical approaches in speech and \\ 2 language neuroscience
}

3 Sophie Bouton ${ }^{1 *}$, Valérian Chambon ${ }^{2 *}$, Narly Golestani ${ }^{3}$, Elia Formisano ${ }^{4,5,6}$ and

$4 \quad$ Anne-Lise Giraud ${ }^{7}$

5 1. Laboratoire Dynamique du Langage, CNRS/Université de Lyon UMR 5596, Lyon, $6 \quad 69007$, France.

7 2. Institut Jean Nicod, CNRS/École Normale Supérieure UMR 8129, PSL University, Paris, $8 \quad 75005$, France.

9 3. Department of Psychology, University of Geneva - Campus Biotech, 9 chemin des 10 Mines, 1202 Geneva, Switzerland.

11 4. Department of Cognitive Neuroscience, Faculty of Psychology and Neuroscience, 12 Maastricht University, Maastricht, the Netherlands.

13 5. Maastricht Brain Imaging Center, Maastricht, the Netherlands.

14 6. Maastricht Centre for Systems Biology, Maastricht University, Maastricht, the $15 \quad$ Netherlands

16 7. Department of Neuroscience, University of Geneva - Campus Biotech, 9 chemin des 17 Mines, 1202 Geneva, Switzerland.

18 * These authors contributed equally to this work.

19 Correspondence: anne-lise.giraud@unige.ch (Anne-Lise Giraud)

20 Keywords. Statistics, Machine Learning, General Linear Model, Interpretation, Speech, 21 Language. 
Abstract

Brain functions are traditionally investigated using general linear models (GLM) in controlled experiments, which allow for a straightforward interpretation of the data. However, constraints imposed by controlled experimental setups have led neuroscientists to increasingly use machine-learning inspired approaches, which allow to work with large datasets collected in relatively natural experimental settings. Given the disparity of available methods and underlying concepts, synthesizing and relating the results of both traditional and machine-learning inspired experiments represents a substantial challenge. In this review, we propose to use a taxonomy based on the spatial specificity of the available methods and the extent to which they are constrained by a priori hypotheses, to classify their interpretability. Taking speech processing as a case example, we dissect the advantages and limitations of each of these methodological approaches, and highlight the ways in which they can be fruitfully combined to facilitate data interpretation.

/main text

The diversity of statistical approaches in cognitive neuroscience

A common experimental paradigm in cognitive neuroscience is to measure changes in neural activity in response to controlled manipulations of stimuli, tasks or behaviours. In these experiments, measured neural responses are generally analysed using a variety of statistical tests (e.g. t-test, F-tests, ANOVA) that are special cases of the so-called General Linear Model (GLM). These statistical analyses relate, at each point in anatomical space, the variance of neural responses with a set of pre-specified variables (predictors) which model the hypothesized effects of experimental manipulations and possible confounding factors. The GLM-based approach is well suited to test pre-defined hypotheses on the relation between specific components of cognitive and psychological models and the underlying functional neuroanatomy. In GLMs, the interpretation of the model fit to the data and the contribution of individual predictors is straightforward: predictors of the model are in small number and directly relate to controlled and a priori uncorrelated crafted experimental manipulations. In language research, for example, GLM-based studies have confirmed the important notion that speech and language-related cognitive operations emerge from processing hierarchies where each step has its own anatomofunctional specificity (Ding et al., 2016; Hickok \& Poeppel, 2007; Sheng et al., 2018) (Figure 1, left panel).

Contrasting with this traditional paradigm, a more "naturalistic" approach has gained considerable traction in recent years (Poldrack \& Yarkoni, 2016; Varoquaux \& Poldrack, 2019). In these experiments, participants are presented with "real world" stimuli, with the goal of measuring how the brain responds to e.g. language "as it is used" (Hamilton \& Huth, 2018). Neural responses collected using e.g. movies or audiobooks are often analysed using encoding models. In language research, encoding models have for example revealed semantic-category selective organization within broadly distributed brain regions ((Huth, de Heer, et al., 2016), Figure 1, middle panel). Similarly to GLMs, encoding models fit the neural responses univariately (i.e., voxel-by-voxel), with the goal of explaining/predicting the level of brain activity through the (linear) combination of a set of variables - typically, features of the stimuli. Interpreting the results of encoding 
models is therefore more difficult than interpreting those of GLMs because the influence of each feature cannot be singled out, as the number of features might be large (in relation to the dataset), and the features might be correlated among them. This implies the use of various techniques (e.g. regularization, cross-validation) that further influence the estimated contribution of individual variables to the overall fitting. The interpretation of encoding analyses can hence benefit from comparing different stimulus representation models, with the assumption that the winning model reflects the actual neural representation (Santoro et al., 2014).

Unlike encoding models, decoding methods exploit the information in spatial patterns of neural responses and can be used to predict perception or behaviour (e.g. motor, speech output) or reconstruct the stimuli using the neural activity itself (Holdgraf et al., 2017). Decoding analyses rely on diverse machine learning techniques and can be broken down into (1) discriminative methods (e.g. classifiers), which aim at detecting multivariate differences between predefined task conditions or stimulus features (within small sets of voxels, regions, or the whole brain), and (2) model-based methods, which aim at reconstructing new stimuli or behaviours that are not present in the training set from brain activity, by predicting features of otherwise well-specified computational models, as in encoding (King et al., 2018). Note that encoding models can be used in conjunction with decoding models, often to optimize the performance of the latter (see (Naselaris et al., 2011, 2015), Figure 1, right panel). In language research, a recent study leveraged a model of the vocal system to decode the speech signal from the brain's response, making significant progress towards the possibility of restoring spoken communication in patients with paralysing conditions (Anumanchipalli et al., 2019).

Several high-profile studies have recently highlighted the value of unsupervised clustering methods - e.g., dimensionality reduction techniques. Their most defining principle is to relax the constraints imposed by supervised, model-based approaches such as encoding/decoding. This allows a maximum of variance (or information) in the data to be accounted for by a combination of $n$ non pre-specified dimensions (Brunton \& Beyeler, 2019; Jack et al., 2018). Importantly, these unsupervised, data-driven methods (e.g. principal component analysis, PCA) do not use labels or specific information regarding the experimental design. Although these methods are often applied to conduct exploratory analyses on large data sets, they are also frequently used with the ambitious goal of discovering unpredicted representational and/or coding principles in the brain. This raises a number of issues that we attempt to expose in this article (see Behavioural relevance and functional specialization). 


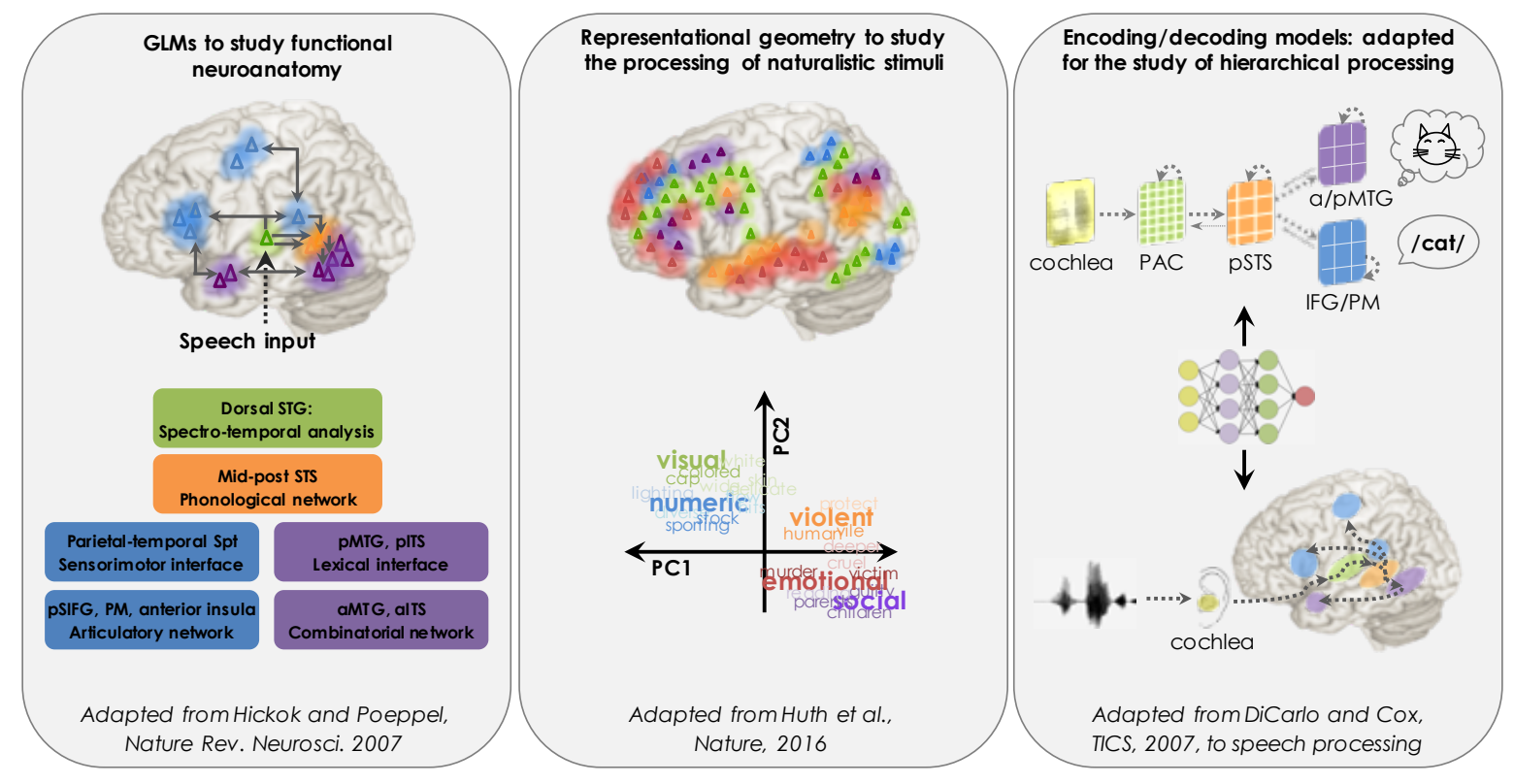

Figure 1. Different approaches for studying brain functional organisation. Left panel: General linear models (GLMs): Traditional approaches using GLMs combined with controlled experiments have revealed a neural architecture where low-level features (i.e., spectro-temporal cues) are transformed along the cortical hierarchy to incrementally construct abstract linguistic structures. Figure adapted from Hickok \& Poeppel, 2007. Middle panel: Representational Geometry: Data-driven approaches for the analysis of large numbers of semantic features in naturalistic stimuli have revealed a semantic-category selective organization that is distributed broadly across brain regions. Figure adapted from Huth et al., 2016. Right panel: Encoding/decoding models: The open-ended interpretation space of encoding or decoding methods can be constrained by using designs that are adapted to the known functional hierarchy of the speech and language system. Figure adapted from DiCarlo \& Cox, 2007 to speech processing.

With the increasing variety of data analysis methods in speech and language neuroscience, making sense of the findings has become a priority. In this paper, we present an operational taxonomy that classifies statistical approaches according to their interpretability, which we define along two dimensions: 1) the spatial (anatomical) specificity of the approach being chosen, and 2) the extent to which data collection and analysis are constrained by pre-defined hypotheses (data driven vs. hypothesis driven analyses) (Figure 2). Relative to traditional univariate approaches, the strength of machine-learning multivariate methods lies in the fact that they can reveal novel organisational principles in large data sets, such as complex geometric or n-dimensional representational patterns (Chartier et al., 2018). Yet, sensitivity to complex patterns comes at the cost of interpretability: understanding the contribution of a single voxel to distributed/multidimensional patterns of brain activity is a difficult and not yet surmounted challenge (Hebart \& Baker, 2017; Ritchie et al., 2017). Moreover, the extent to which machine-learning inspired approaches are constrained by a priori hypotheses influences the level of specification of model features, which, in turn, determines how straightforward 
the interpretation of results can be. In this review, we use this taxonomy to show that the difference in spatial specificity between methods, as well as the relative absence of biologically informed hypotheses, can lead to different interpretations of findings. We then describe different existing ways in which analysis approaches can be optimised to allow for more constrained interpretations of cognitive neuroscience research in the domain of speech and language, while at the same time capitalizing on the ability to exploit multivariate differences in the real world and/or uncover unpredicted organising neural principles.

\section{Figure 2}

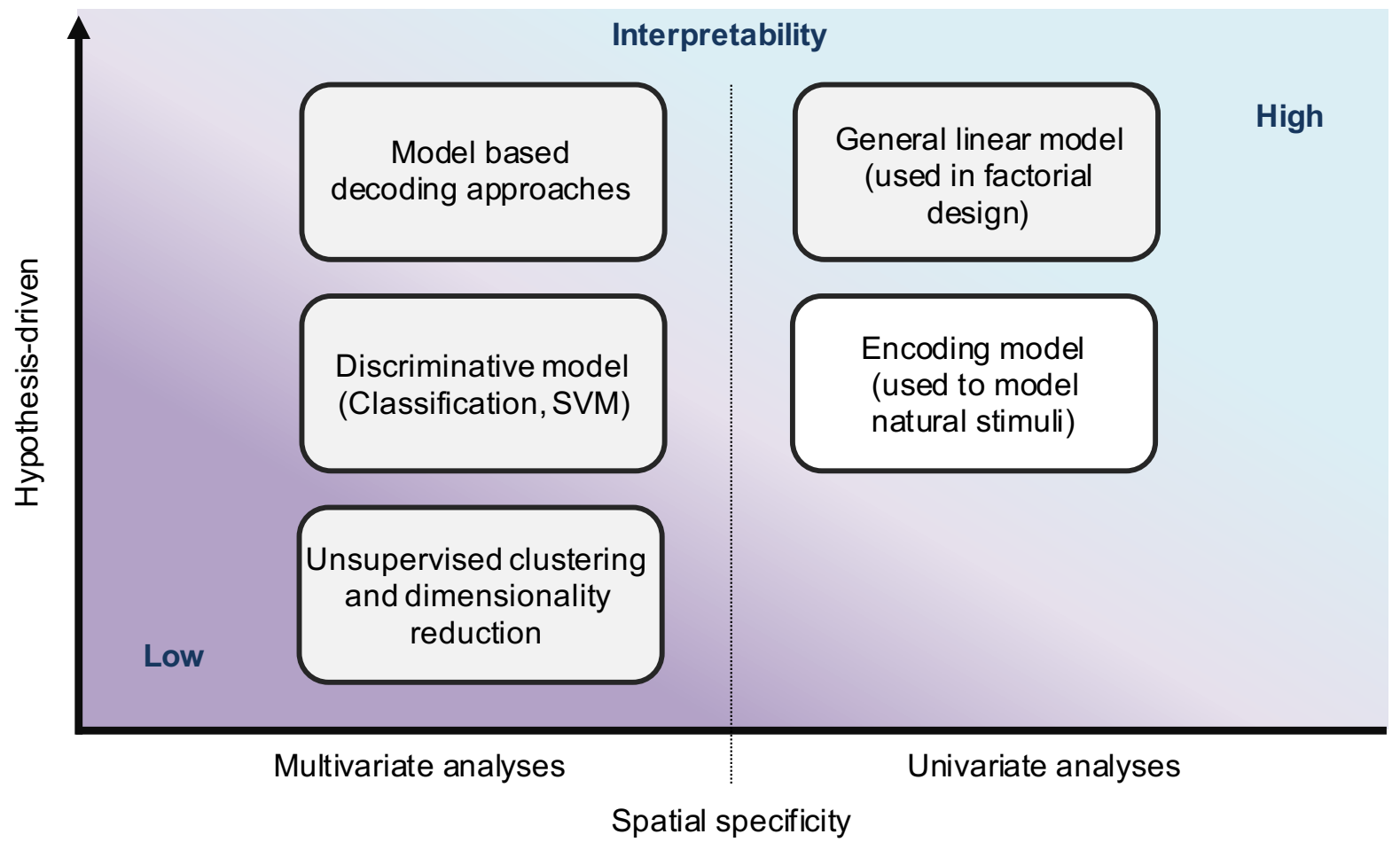

Figure 2. A proposed taxonomy of statistical approaches/methods in cognitive neuroscience. The classification is based on two dimensions: (i) the "hypothesis-driven" dimension, which is characterised by a continuum between hypothesis- and data-driven approaches, i.e. between analysis methods that are to a greater or lesser extent guided by predefined assumptions (note that "data-driven" does not necessarily equate to "theory-free"); (ii) the spatial (anatomical) specificity of the approach: a univariate model considers a single spatial point at a time, whereas a multivariate model considers many spatial points at once, making the anatomical parcellation/interpretation of neural patterns more difficult.

\section{Behavioural relevance and functional specialisation}

Considerable efforts have been made in recent years to better understand how the brain forms concepts based on word meaning. One proposal is that cortical regions are organized in large-scale networks which both underpin semantic relations and support the emergence of higher-level concepts (Hagoort, 2014). Accordingly, and in parallel to classical hypothesis-testing experimental designs (Maess et al., 2016; Teige et al., 2018), studies using machine-learning inspired approaches have revealed a semantic-category 
154 organization that is broadly distributed across the whole brain (Huth et al., 2012; Huth, de 155 Heer, et al., 2016; Y. Zhang et al., 2020). Here, to characterize the semantic selectivity of

156 each voxel, neural activity was measured with fMRI while participants passively listened to 157 long narratives or watched natural movies. Neural activity parameters were fitted to 158 semantic features using univariate linear regression (voxel-wise modelling), and the fitted 159 model was projected onto a low-dimensional subspace obtained by applying 160 dimensionality reduction techniques (e.g. principal component analysis, PCA). The 161 resulting four dimensions were those that best explained the data. Importantly, no explicit 162 assumptions were made regarding the combination of features that these four dimensions 163 reflected. However, this raises some concerns related to the interpretation of the results. 164 The inclusion of many independent variables (i.e., semantic features) requires strong post165 hoc assumptions about the latent/underlying organisation of the resulting neural patterns, 166 and some implicit assumptions might be incorrect. For instance, most studies implicitly 167 assume that neuronal responses from any location in the brain can encode any 168 representative semantic class, and that they do so based on locally specific functional 169 recruitment patterns. Ideally, testing the specificity of these patterns would require to 170 decode the same semantic features from neural activity at the same locations. Subsequent 171 studies using such decoding methods, with maps based on the weight distribution across 172 semantic labels, revealed less local semantic category specificity than expected (Huth, 173 Lee, et al., 2016; Pereira et al., 2018). In these follow-up studies, each voxel of a given 174 semantic map was associated with the meaning of few but semantically related words, 175 suggesting a global 'map' of semantic categories with a broad distribution of semantic 176 knowledge in the brain. It remains difficult, however, to reconcile these essentially 177 descriptive conclusions with hierarchical processing where auditory input is progressively 178 abstracted into higher-level language representations. The recent mapping of hidden 179 units of deep architecture models with neuroimaging data speaks to such progressive 180 abstraction process in natural language processing (Kell et al., 2018).

\section{The multiple interpretations of data-driven dimensions}

182 Finding out what information is contained in multivariate patterns arising from data-driven 183 models is a necessary yet difficult task. Interpreting how neural data maps onto 184 behavioural data or stimuli in an n-dimensional space requires dimensionality-reduction, 185 which in turn leads to arbitrary interpretations since it relies on post-hoc interpretations 186 regarding what each eigenvariate may represent. If no explicit hypothesis is formulated 187 during the reduction of observed dimensions (i.e., during unsupervised procedures), the 188 interpretation is uncertain. Each eigenvariate resulting from dimensionality reduction 189 procedures describes a complex trajectory in a 4-dimensional (i.e., including time) state190 space. Understanding what these trajectories represent and how they relate to the 191 functional processing of specific regions is ultimately the hardest problem. If such 192 trajectories are specific to functional regions, they might reveal the 193 complexity/polyvalence of neural population coding, depending on task requirements 194 (Rubin et al., 2019). However, if the sampled regions are ill-defined, e.g. when they are 195 arbitrarily determined by cortical electrode grids or arrays used in the measurements, 
these trajectories might reflect the mixed contribution of different computational levels resulting from multiple regions within the hierarchy (Roussel et al., 2020).

This point is well illustrated by a specific example in the speech literature. Using a data-driven approach (i.e., principal component and clustering analyses), Bouchard and colleagues identified patterns of spatially distributed neural activity in the human sensorimotor cortex during the production of consonant-vowel syllables (Bouchard et al., 2013). The authors proposed that these patterns reflect articulatory features, i.e., elementary speech motor sequences. Although this interpretation is reasonable, one may argue that an equally valid interpretation of the results is an organization that follows acoustic traits. The phonemic distribution in a 2D state-space highlighted by Bouchard (Linear Discriminate Analysis (LDA), Suppl. Fig. 10 in (Bouchard et al., 2013), Figure 3A) aligns surprisingly well with the spatial distribution of acoustic features described in 1969 by Jakobson, Fant, \& Halle (pp. 33-348; see also Figure 3B, where consonants and vowels cluster along low/acute (LDA1) and compact/diffuse (LDA2) continua). Given the pivotal role of the somatosensory system in the hierarchical organisation of speech production (Franken et al., 2018), an organization based on combined acoustic and articulatory features provides the most likely interpretation for the phonemic topography emerging from neural activity in the ventral sensorimotor cortex (vSMC). This example illustrates that the same data-driven analysis can lead to very different interpretations, and that the conclusions that are drawn depend on the models that the experimenters have in mind and on the context in which results are interpreted (Bzdok et al., 2018). Unlike the hypothesis testing approach, the data-driven approach used in isolation ultimately leads to untested interpretations. In summary, and although it may appear counter-intuitive, the use of data-driven models requires extremely solid background hypotheses for post-hoc interpretation.

Can the brain exploit multidimensional features?

The choice of machine learning approaches in cognitive neuroscience implicitly contains the fundamental assumption that a given brain area can represent multiple features, and that these features can be captured by multivariate patterns. Yet, multivariate patterns may also capture phenomena other than locally processed features, such as, in particular, properties that are inherited from distant regions with which the sampled brain region interacts. For example, in patients with intracortical electrodes, it has been shown that the activity pattern identified in the ventral sensorimotor cortex (VSMC) corresponds to different articulatory features when the patients produced versus when they listened to syllables (Cheung et al., 2016). The authors argued that the neural response structure in the vSMC is organized according to "place of articulation" during speech production, but according to "manner of articulation" during speech listening, while the neural response in the STG is organized according to "manner of articulation" while both speaking and listening (Mesgarani et al., 2014) (Figure 3C and 3D). When speech is perceived and then produced, the conversion of acoustic cues into motor routines involves the VSMC, which interfaces auditory and motor cortices (Assaneo \& Poeppel, 2018; Liebenthal \& Möttönen, 2017; Pulvermüller \& Fadiga, 2010). Thus, we could argue here that the fact that articulatory features can be decoded similarly within the vSMC and the STG does not 
unambiguously demonstrate that these regions encode or represent articulatory categories, but could equally mean that the structure of the articulatory space shares common properties with that of the acoustic space (see (Popov et al., 2018) for a similar point about shared variance in non-equivalent representational spaces). In principle, this isomorphism can be exploited by any "information-greedy" decoder to retrieve, in one representational space, the features that in fact only reflect meaningful dimensions in a different space. In this example, articulatory representations can be classified in a region known to encode acoustic features (the STG) simply because there is an (even partial) mapping between the acoustic and articulatory representational schemes, which causes their intrinsic representational properties to be correlated (Assaneo \& Poeppel, 2018).

\section{Figure 3}

(a)

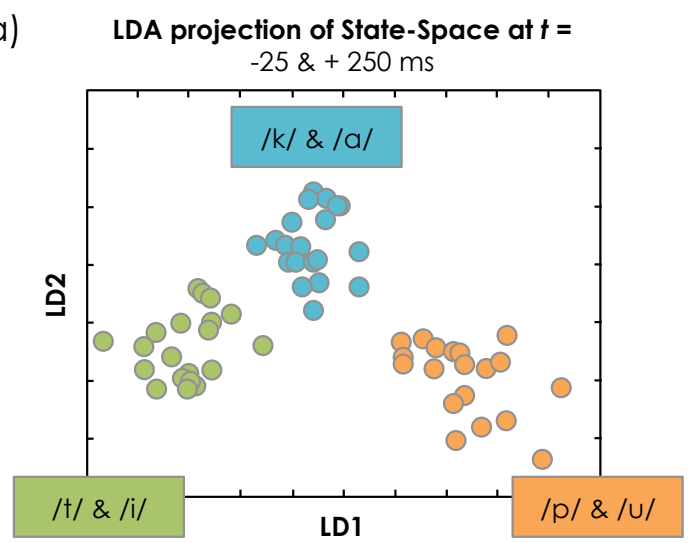

(c)
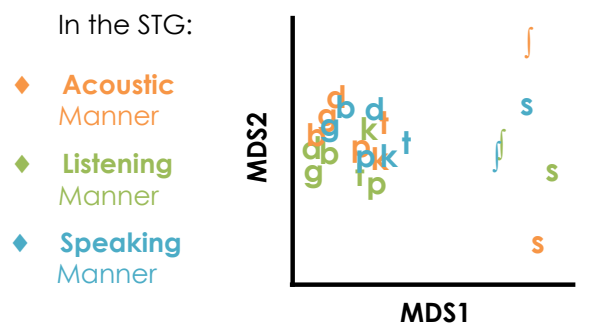

(b) Spatial distribution of acoustic features (Jakobson, 1969)

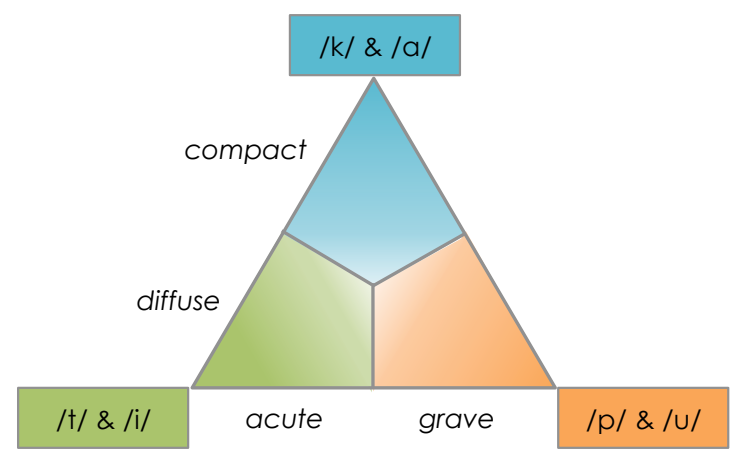

(d)

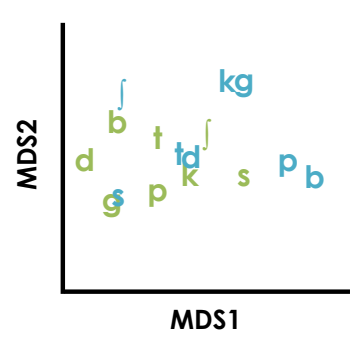

Figure 3. Differing interpretations depending on whether data collection is constrained by prior theoretically or biologically informed hypotheses. (a) and (b): comparison between the discriminant analyses in Bouchard et al., 2013 (Bouchard et al., 2013) with the acoustic features of Jakobson et al., 1969 (Jakobson et al., 1961). Bouchard et al. (2013) propose that these patterns (a) reflect articulatory features (i.e., elementary speech motor sequences), but an organization according to acoustic features (b) also provides a plausible interpretation of the findings. Images 3a \& 3b courtesy of Michel Hoen and Willy Serniclaes. (c) In the STG (superior temporal gyrus), decoding patterns arising from machine learning algorithms show that the dimensions distinguishing the neural responses during listening and speaking are organized according to 'manner of articulation' cues. (d) In the VSMC (ventral sensorimotor cortex), decoding patterns show that neural responses are organized according to 'manner of articulation' cues during speech listening and according to 'place of articulation' cues when speaking. Fig. 3a adapted from 
Bouchard et al., Suppl. Fig. 10, and Fig. 3b adapted from Jakobson et al., 1969, Figs. pp. 33-34.

264 Figures 3c \& 3d adapted from Cheung et al., 2016 (Cheung et al., 2016), Figure 4 and Suppl. 2 265 Fig.

\section{Optimizing multivariate analysis methods}

Given the exploratory potential and also the interpretability issues of multivariate, machine-learning inspired approaches in cognitive neuroscience (Varoquaux \& Thirion, 2014), it seems important to think about ways in which such methods can be capitalized on while at the same time limiting some of their pitfalls. One way to tackle this is to prioritize the use of model-based approaches, and as such to make them relatively more hypothesis-driven (De Angelis et al., 2017; King \& Dehaene, 2014; Moses et al., 2016; Pasley et al., 2012). Furthermore, in particular in the domain of speech and language neuroscience, these models could be specifically tailored to the level(s) of the processing hierarchy and/or of the functional role of the region(s) being examined (Çelik et al., 2019; Deniz et al., 2019; Rutten et al., 2019; Venezia et al., 2019). Second, depending on the goal of the investigation, multiple models can be combined in order to question different levels of processing simultaneously (Anumanchipalli et al., 2019; de Heer et al., 2017), or applied separately to different functional subregions, based on the a priori hypotheses and predictions of the study (Kell et al., 2018). Third, again especially in speech and language neuroscience, it is primordial that modelling approaches take into account the dynamics of the signal, thereby constraining the search and thus also the interpretation space of machine learning approaches, this time in the temporal domain (Dichter et al., 2016; King \& Dehaene, 2014; Loula et al., 2018; Moses et al., 2016).

We hence cautiously advocate that relating the output of multivariate analysis methods (i.e., multivariate patterns, state-space representations or classifier performance) to behaviour ought to involve the specification of tailored computation rules. For each possible task, and at each level of the processing hierarchy, there are optimal preinformed ways of mapping the neural activity according to the variable of interest (Issa et al., 2018; Santoro et al., 2017). Known features of the functional hierarchy in the speech processing system could help to define an optimal algorithm, or a time-resolved model that varies depending on the processing stage (Figure 4). In visual perception, for instance, a geometric model can be defined whose features adapt to the known computational properties of each level of the functional hierarchy, from specific to general as one goes up towards more abstract levels (DiCarlo \& Cox, 2007; Grill-Spector \& Weiner, 2014; Nunez-Elizalde et al., 2018; van Gerven, 2017). For speech processing, to recover the function of the first two levels of the auditory hierarchy (Jiang et al., 2016; Santoro et al., 2017), it seems necessary to use models that take both the acoustic spectrum and time into account, with a spectro-temporal receptive field (STRF) model being more accurate in primary auditory cortex, and an STRF model tailored to phonological cues being more accurate in auditory association cortex (Dewitt \& Rauschecker, 2012; Venezia et al., 2019). The same strategy may apply to higher-order regions where the natural dimensions of the feature space are less well known, i.e., semantic regions. At these levels of the processing hierarchy, linguistic representations are presumably a-temporal, encoded with sparse or rate codes (Chang et al., 2010; 


\section{Figure 4}

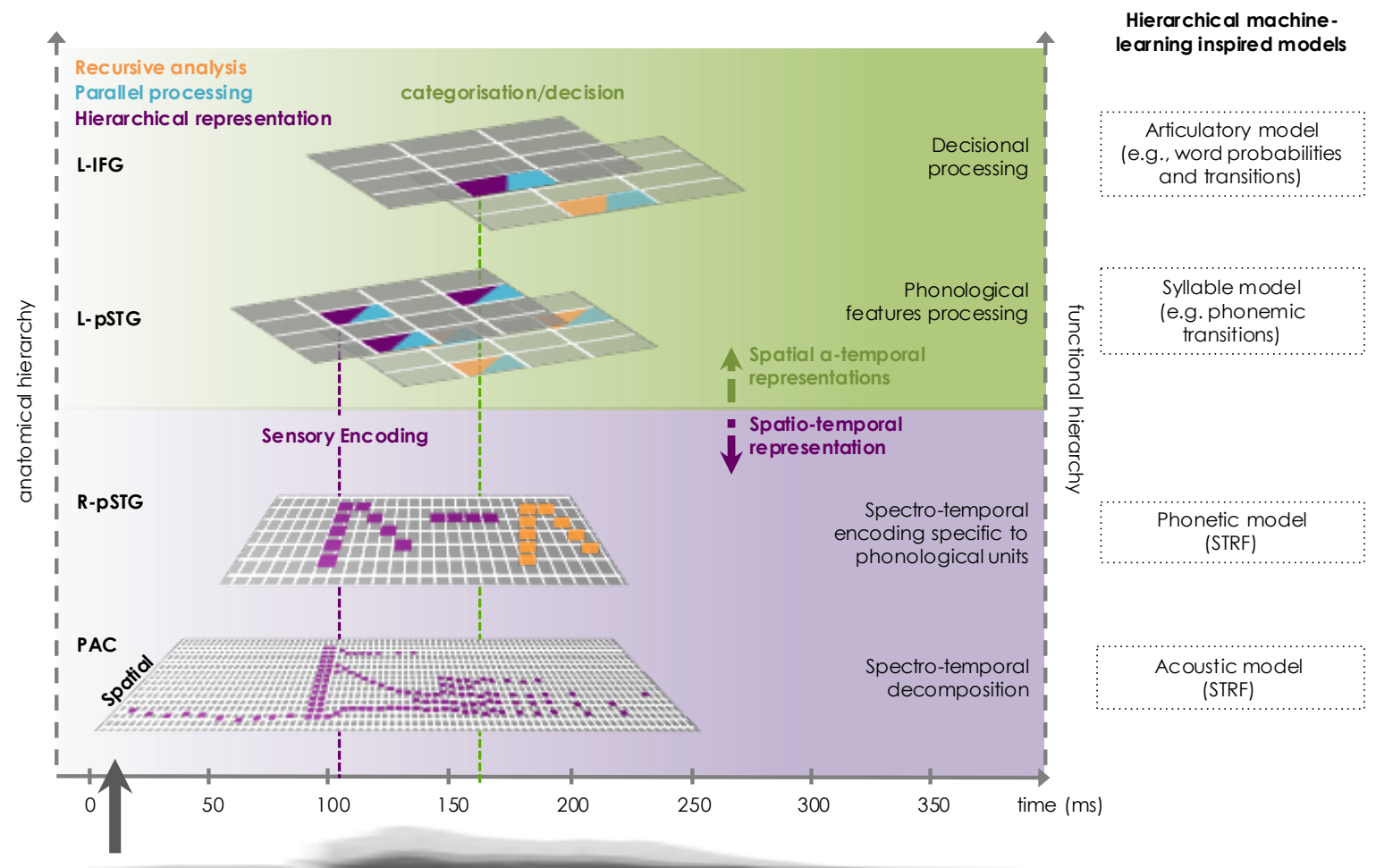

Figure 4. The importance of non-linear encoding in speech perception. Here we consider four processing stages in a very simple /ba/- /da/ categorisation task, each of them relying on the activation of a circumscribed area underlying a specific function. Along the processing hierarchy, time-based representations are gradually abstracted, from fine spectro-temporal decomposition in primary auditory cortex (PAC), to spectro-temporal encoding specific to phonological units in bilateral PSTG (posterior superior temporal gyrus), and phonological feature processing in the left PSTG, and finally to decisional processing in the left inferior frontal gyrus (IFG) (Bouton et al., 2018). Along the hierarchy, information is encoded through hierarchical, parallel and recursive processes (MacGregor et al., 2012; Strijkers, 2016). Representations are encoded within different time frames in the right and left PSTG; this is important to consider when using classification methods. A way to use machine-learning inspired methods within a hypothesis-driven approach would be, for example, to run multivariate-pattern and other forms of machine learning analyses on different types of measures selected based on the functional region they originate from, i.e., using STRFs at the lower levels of the auditory hierarchy (Rutten et al., 2019; Santoro et al., 2017), phonemic transitions in the left PSTG or word probabilities and transitions in the left IFG.

Such model-based decoding has been used to study how the auditory encoding of different acoustic dimensions is modified depending on whether people attend to linguistic (e.g. phonological) or para-linguistic (i.e. voice) information in pseudo-words

329 (Rutten et al., 2019). Although in this study only an acoustic model was used for 330 questioning the data, extensions to models containing e.g. pitch and higher level 
phonemic features would allow to generate an 'embedded', or concatenated model space (de Heer et al., 2017). Here, it might be expected that different features (e.g., acoustic, phonological and pitch) best explain the neural data within different functional subregions of the auditory hierarchy and/or during different tasks, allowing to gain a broader understanding of how our auditory system makes use of linguistic and paralinguistic information in speech. Two recent fMRI studies having compared the performance of models targeting different processing levels (i.e. acoustic versus pitch (De Angelis et al., 2017) and spectral, articulatory and semantic (de Heer et al., 2017) illustrate how model-based machine learning approaches can be used to tap into different levels of the processing hierarchy, and to complement hypothesis driven approaches in revealing the regional functional specificity of these.

Approaches that deviate from the GLM experimental approach can be more finetuned for research on the neuroscience of language not only by adapting models to assess different, regionally specific levels of processing within the language processing hierarchy, but also by fine-tuning models at different levels of temporal resolution depending on the processing level within this hierarchy. Several recent ECoG studies illustrate the importance of explicitly accounting for the dynamics of the system (Dichter et al., 2016; Moses et al., 2016; Pasley et al., 2012). The challenge for future work will be to extend these approaches within the context of language neuroscience research to noninvasive, whole-brain, ideally time-resolved studies (Gwilliams \& King, 2020; Loula et al., 2018). This would allow to elaborate language processing models with respect to temporal dependencies, and to reveal how these are gradually abstracted across different levels of the processing hierarchy across the brain.

In vision, a field where data-driven methods have led to more easily interpretable results than in the auditory domain, several research groups propose to use detailed hierarchical artificial neural network models to address neural information transformation processes along the visual pathways (Cichy et al., 2014; Mohsenzadeh et al., 2019). Bashivan et al. showed that images synthesized by artificial neural networks can precisely and selectively activate neural populations in specific regions of the monkey visual system 360 (Bashivan et al., 2019). Such tools can also be used in a powerful way in the field of speech and audition, as artificial neural networks can decode, with high precision, the latent structure learned from single trials, revealing a rich, hierarchical organization of linguistic features (Livezey et al., 2019). These examples illustrate how the reverse engineering of artificial neural networks can allow to gain a deep understanding of neural processes underlying complex cognitive functions (Kell et al., 2018; Saxe et al., 2021).

Can we get rid of behaviour?

Drawing on unsupervised sorting of mouse neuronal activity, Rubin and colleagues found that neurons could be classified based on their internal tuning properties rather than on external variables such as a pre-defined stimulus (Rubin et al., 2019). The technique used by the authors, i.e., non-linear dimensionality reduction, has some definite advantages

371 since it can reveal informative patterns of neural activity that are not tied to a particular 372 model of the stimulus set (Zylberberg, 2018). This study suggests that one could 373 investigate brain circuits for which little is known about the identity of the variables they 
encode, in the same way that dimensionality reduction can be leveraged for probing the representational space of brain regions for which the natural dimensions of the feature space are unknown, these primarily being high-order associative regions. Whether we can truly get rid of any behavioural benchmark using multivariate pattern analyses or other machine learning methods, and whether we can elicit or modify specific behaviours by artificially inducing multivariate patterns (e.g. using stimulation techniques or brain computer interfaces (Ezzyat et al., 2017; Shibata et al., 2019)) are primordial issues for future research (Grootswagers et al., 2018; Krakauer et al., 2017; Panzeri et al., 2017).

\section{Conclusion}

383 With examples from the speech and language literature, we illustrate strengths and weaknesses of using machine-learning inspired methods as alternatives to classical GLM methods. The fact that machine-learning inspired methods impose little constraint on how information is selected and classified, and on how dimensions are extracted, allows to reveal unexpected structure in the data, for example in the form of multidimensional, spatially distributed representations. But this higher sensitivity to non-predicted dimensions may come at the cost of not seeing the true structure that the underlying neuronal populations represent, and hence, of missing dimensions in the data that truly relate to the observed behaviour (Carlson et al., 2017). Addressing these specific concerns may require the use of adjusted methods, which take advantage of biologically informed priors so as to functionally limit the size of the model space (Ahlheim \& Love, 2018; Snoek et al., 2019).

In the era of big data, it is now commonplace to say that scientists need theory more than ever. Two decades ago, science-fiction writer Ted Chiang wrote a premonitory article in Nature purporting to celebrate the 25th anniversary of the last human scientific publication (Chiang, 2000). In this provocative, futuristic short-story, science is now exclusively conducted by genetically improved humans, called "metahumans", and all that remains for human scientists is the interpretation of metahuman data. "In the face of metahuman science, humans have become metascientists". It is of course tempting to see metahuman science as a metaphor for the use of artificial intelligence methods, whose inner functioning and scientific output sometimes appear to lie beyond the reach of our understanding. But data are rarely, if ever, self-explanatory. They make sense to the extent that they validate or falsify theoretically informed predictions. When it comes to interpreting data-driven findings, possessing a biologically informed theory beforehand retains some usefulness. "Human researchers", says Chiang, "may discern applications overlooked by metahumans, whose advantages tend to make them unaware of our concerns". The same surely applies to data-driven methods, whose appeal and 


\section{Acknowledgments}

413 We wish to thank Willy Serniclaes and Michel Hoen for their contribution to the critical 414 review of some of the work discussed here, and Timothée Proix for his helpful comments 415 and suggestions on an earlier draft of this article. This work was funded by the Swiss 416 National Science Foundation (SNF 320030_149319 and SNF 320030_163040 to A-L.G., 417 SNF P300P1_167591 to S.B., and SNF 100014_182381 to N.G.), by the Fondation pour 418 I'Audition (RD-2016-5 to S.B.), by the Fyssen Foundation (to S.B.), by the Agence 419 Nationale pour la Recherche (ANR, Grants ANR-17-EURE-0017 FrontCog, ANR-10-IDEX420 0001-02 PSL* and ANR-16-CE37-0012-01 to V.C.), and by the University of Geneva 421 Language and Communication Research Network (FID-LC-EXPERTSE to N.G.). EF is 422 partially supported by the Dutch Province of Limburg. 
424

425

426

427

428

429

430

431

432

433

434

435

436

437

438

439

440

441

442

443

444

445

446

447

448

449

450

451

452

453

454

Ahlheim, C., \& Love, B. C. (2018). Estimating the functional dimensionality of neural representations. NeuroImage, 179, 51-62. https://doi.org/10.1101/232454

Anumanchipalli, G. K., Chartier, J., \& Chang, E. F. (2019). Speech synthesis from neural decoding of spoken sentences. Nature, 568(7753), 493-498. https://doi.org/10.1038/s41586-019-1119-1

Assaneo, M. F., \& Poeppel, D. (2018). The coupling between auditory and motor cortices is rate-restricted: Evidence for an intrinsic speech-motor rhythm. Science Advances, 4(2), 1-10. https://doi.org/10.1126/sciadv.aao3842

Bashivan, P., Kar, K., \& DiCarlo, J. J. (2019). Neural population control via deep image synthesis. Science, 364(6439), eaav9436. https://doi.org/10.1126/science.aav9436

Bouchard, K. E., Mesgarani, N., Johnson, K., \& Chang, E. F. (2013). Functional organization of human sensorimotor cortex for speech articulation. Nature, 495(7441), 327-332. https://doi.org/10.1038/nature11911

Bouton, S., Chambon, V., Tyrand, R., Guggisberg, A. G., Seeck, M., Karkar, S., Ville, D. Van De, \& Giraud, A.-L. (2018). Focal versus distributed temporal cortex activity for speech sound category assignment. PNAS, 115(6), E1299-E1308. https://doi.org/https://doi.org/10.1101/133272

Brunton, B. W., \& Beyeler, M. (2019). Data-driven models in human neuroscience and neuroengineering. Current Opinion in Neurobiology, 58, 21-29. https://doi.org/10.1016/j.conb.2019.06.008

Bzdok, D., Altman, N., \& Krzynwinski, M. (2018). Statistics versus machine learning. Nature Methods, 15(4), 233-234. https://doi.org/10.1038/nmeth.4642.Statistics

Carlson, T. A., Goddard, E., Kaplan, D. M., Klein, C., \& Ritchie, J. B. (2017). Ghosts in machine learning for cognitive neuroscience: Moving from data to theory. NeuroImage, 180, 88-100. https://doi.org/10.1016/j.neuroimage.2017.08.019

Çelik, E., Dar, S. U. H., Yılmaz, Ö., Keleş, Ü., \& Çukur, T. (2019). Spatially informed voxelwise modeling for naturalistic fMRI experiments. NeuroImage, 186, 741-757. https://doi.org/10.1016/j.neuroimage.2018.11.044

Chang, E. F., Rieger, J. W., Johnson, K., Berger, M. S., Barbaro, N. M., \& Knight, R. T. (2010). Categorical Speech Representation in the Human Superior Temporal Gyrus. Nature Neuroscience, 13(11), 1428-1432. https://doi.org/10.1038/nn.2641.Categorical 
455

456

457

458

459

460

461

462

463

464

465

466

467

468

469

470

471

472

473

474

475

476

477

478

479

480

481

482

483

484

485

486

487

Chartier, J., Anumanchipalli, G. K., Johnson, K., \& Chang, E. F. (2018). Encoding of Articulatory Kinematic Trajectories in Human Speech Sensorimotor Cortex. Neuron, 98(5), 1042-1054. https://doi.org/10.1016/j.neuron.2018.04.031

Cheung, C., Hamilton, L. S., Johnson, K., \& Chang, E. F. (2016). The auditory representation of speech sounds in human motor cortex. ELife, 5, e12577. https://doi.org/10.7554/eLife.12577

Chiang, T. (2000). Catching crumbs from the table. Nature, 405, 517.

Cichy, R. M., Pantazis, D., \& Oliva, A. (2014). Resolving human object recognition in space and time. Nature Neuroscience, 17(3), 1-10. https://doi.org/10.1038/nn.3635

De Angelis, V., De Martino, F., Moerel, M., Santoro, R., Hausfeld, L., \& Formisano, E. (2017). Cortical processing of pitch: Model-based encoding and decoding of auditory fMRI responses to real-life sounds. NeuroImage, 180, 291-300. https://doi.org/10.1016/j.neuroimage.2017.11.020

de Heer, W. A., Huth, A. G., Griffiths, T. L., Gallant, J. L., \& Theunissen, F. E. (2017). The hierarchical cortical organization of human speech processing. Journal of Neuroscience, 37(27), 6539-6557. https://doi.org/10.1523/JNEUROSCI.326716.2017

Deniz, F., Nunez-Elizalde, A. O., Huth, A. G., \& Gallant, J. L. (2019). The representation of semantic information across human cerebral cortex during listening versus reading is invariant to stimulus modality. The Journal of Neuroscience, 39(39), 7722-7736. https://doi.org/10.1523/jneurosci.0675-19.2019

Dewitt, I., \& Rauschecker, J. P. (2012). Phoneme and word recognition in the auditory ventral stream. PNAS, 109(8), 505-514. https://doi.org/10.1073/pnas.1113427109

DiCarlo, J. J., \& Cox, D. D. (2007). Untangling invariant object recognition. Trends in Cognitive Sciences, 11(8), 333-341. https://doi.org/10.1016/j.tics.2007.06.010

Dichter, B. K., Bouchard, K. E., \& Chang, E. F. (2016). Dynamic Structure of Neural Variability in the Cortical Representation of Speech Sounds. Journal of Neuroscience, 36(28), 7453-7463. https://doi.org/10.1523/JNEUROSCI.0156-16.2016

Ding, N., Melloni, L., Zhang, H., Tian, X., \& Poeppel, D. (2016). Cortical tracking of hierarchical linguistic structures in connected speech. Nature Neuroscience, 19(1), 158-164. https://doi.org/10.1038/nn.4186

Ezzyat, Y., Kragel, J. E., Burke, J. F., Levy, D. F., Lyalenko, A., Wanda, P., Sullivan, L. O., Hurley, K. B., Busygin, S., Pedisich, I., Sperling, M. R., Worrell, G. A., Kucewicz, M. T., 

Davis, K. A., Lucas, T. H., Inman, C. S., Lega, B. C., Jobst, B. C., Sheth, S. A., ... Kahana, M. J. (2017). Direct Brain Stimulation Modulates Encoding States Article Direct Brain Stimulation Modulates Encoding States and Memory Performance in Humans. Current Biology, 27(9), 1251-1258. https://doi.org/10.1016/j.cub.2017.03.028

Franken, M. K., Acheson, D. J., McQueen, J. M., Hagoort, P., \& Eisner, F. (2018). Opposing and following responses in sensorimotor speech control: Why responses go both ways. Psychonomic Bulletin \& Review, 25(4), 1458-1467. https://doi.org/10.17605/OSF.IO/DAR54

Grill-Spector, K., \& Weiner, K. S. (2014). The functional architecture of the ventral temporal cortex and its role in categorization. Nature Reviews Neuroscience, 15(8), 536-548. https://doi.org/10.1038/nrn3747

Grootswagers, T., Cichy, R. M., \& Carlson, T. A. (2018). Finding decodable information that can be read out in behaviour. NeuroImage, 179, 252-262. https://doi.org/10.1016/j.neuroimage.2018.06.022

Gwilliams, L., \& King, J.-R. (2020). Recurrent processes support a cascade of hierarchical decisions. ELife, 9, 1-20. https://doi.org/10.7554/eLife.56603

Hagoort, P. (2014). Nodes and networks in the neural architecture for language: Broca's region and beyond. Current Opinion in Neurobiology, 28, 136-141. https://doi.org/10.1016/j.conb.2014.07.013

Hamilton, L. S., \& Huth, A. G. (2018). The revolution will not be controlled: natural stimuli in speech neuroscience. Language, Cognition and Neuroscience, 35(5), 573582. https://doi.org/10.1080/23273798.2018.1499946

Hebart, M. N., \& Baker, C. I. (2017). Deconstructing multivariate decoding for the study of brain function. NeuroImage, 180, 4-18. https://doi.org/10.1016/j.neuroimage.2017.08.005

Hickok, G., \& Poeppel, D. (2007). The cortical organization of speech processing. Nature Reviews. Neuroscience, 8(5), 393-402. https://doi.org/10.1038/nrn2113

Holdgraf, C. R., Rieger, J. W., Micheli, C., Martin, S., Knight, R. T., \& Theunissen, F. E. (2017). Encoding and decoding models in cognitive electrophysiology. Frontiers in Systems Neuroscience, 11, 61. https://doi.org/10.3389/FNSYS.2017.00061 Huth, A. G., de Heer, W. A., Griffiths, T. L., Theunissen, F. E., \& Gallant, J. L. (2016). Natural speech reveals the semantic maps that tile human cerebral cortex. Nature, 532(7600), 453-458. https://doi.org/10.1038/nature17637 
Huth, A. G., Lee, T., Nishimoto, S., Bilenko, N. Y., Vu, A. T., \& Gallant, J. L. (2016). Decoding the semantic content of natural movies from human brain activity. Frontiers in Systems Neuroscience, 10, 81. https://doi.org/10.3389/fnsys.2016.00081

Huth, A. G., Nishimoto, S., Vu, A. T., \& Gallant, J. L. (2012). A Continuous Semantic Space Describes the Representation of Thousands of Object and Action Categories across the Human Brain. Neuron, 76(6), 1210-1224. https://doi.org/10.1016/j.neuron.2012.10.014

Issa, E. B., Cadieu, C. F., \& Dicarlo, J. J. (2018). Neural dynamics at successive stages of the ventral visual stream are consistent with hierarchical error signals. ELife, 7, e42870.

Jack, R. E., Crivelli, C., \& Wheatley, T. (2018). Data-Driven Methods to Diversify Knowledge of Human Psychology. Trends in Cognitive Sciences, 22(1), 1-5. https://doi.org/10.1016/j.tics.2017.10.002

Jakobson, R. C., Fant, G. M., \& Halle, M. (1961). Preliminaries to speech analysis: The distinctive features and their correlates. (MIT Press).

Jiang, W., Pailla, T., Dichter, B., Chang, E. F., \& Gilja, V. (2016). Decoding speech using the timing of neural signal modulation. Proceedings of the Annual International Conference of the IEEE Engineering in Medicine and Biology Society, EMBS, 15321535. https://doi.org/10.1109/EMBC.2016.7591002

Kell, A. J. E., Yamins, D. L. K., Shook, E. N., Norman-Haignere, S. V, \& Mcdermott, J. H. (2018). A Task-Optimized Neural Network Replicates Human Auditory Behavior, Predicts Brain Responses, and Reveals a Cortical Processing Hierarchy. Neuron, 98(3), 630-644. https://doi.org/10.1016/j.neuron.2018.03.044

King, J.-R., \& Dehaene, S. (2014). Characterizing the dynamics of mental representations: the temporal generalization method. Trends in Cognitive Sciences, 18(4), 203-210. https://doi.org/10.1016/j.tics.2014.01.002

King, J.-R., Gwilliams, L., Holdgraf, C. R., Sassenhagen, J., Barachant, A., Engemann, D., Larson, E., \& Gramfort, A. (2018). Encoding and Decoding Neuronal Dynamics : Methodological Framework to Uncover the Algorithms of Cognition. BioRxiv.

Krakauer, J. W., Ghazanfar, A. A., Gomez-Marin, A., MacIver, M. A., \& Poeppel, D. (2017). Neuroscience Needs Behavior: Correcting a Reductionist Bias. Neuron, 93(3), 480490. https://doi.org/10.1016/j.neuron.2016.12.041

Liebenthal, E., \& Möttönen, R. (2017). An interactive model of auditory-motor speech perception. Brain and Language, 187, 33-40. 

https://doi.org/10.1016/j.bandl.2017.12.004

Livezey, J. A., Bouchard, K. E., \& Chang, E. F. (2019). Deep learning as a tool for neural data analysis: Speech classification and cross-frequency coupling in human sensorimotor cortex. PLOS Computational Biology, 15(9), e1007091. https://doi.org/10.1371/journal.pcbi.1007091

Loula, J., Varoquaux, G., \& Thirion, B. (2018). Decoding fMRI activity in the time domain improves classification performance. NeuroImage, 180, 203-210. https://doi.org/10.1016/j.neuroimage.2017.08.018

MacGregor, L. J., Pulvermüller, F., van Casteren, M., \& Shtyrov, Y. (2012). Ultra-rapid access to words in the brain. Nature Communications, 3, 711. https://doi.org/10.1038/ncomms1715

Maess, B., Mamashli, F., Obleser, J., Helle, L., \& Friederici, A. D. (2016). Prediction signatures in the brain: Semantic pre-activation during language comprehension. Frontiers in Human Neuroscience, 10, 1-11. https://doi.org/10.3389/fnhum.2016.00591

Mesgarani, N., Cheung, C., Johnson, K., \& Chang, E. F. (2014). Phonetic feature encoding in human superior temporal gyrus. Science, 343(6174), 1006-1010. https://doi.org/10.1126/science.1245994

Mohsenzadeh, Y., Mullin, C., Lahner, B., Cichy, R., \& Oliva, A. (2019). Reliability and Generalizability of Similarity-Based Fusion of MEG and fMRI Data in Human Ventral and Dorsal Visual Streams. Vision, 3(1), E8. https://doi.org/10.3390/vision3010008

Moses, D. A., Mesgarani, N., Leonard, M. K., \& Chang, E. F. (2016). Neural speech recognition: continuous phoneme decoding using spatiotemporal representations of human cortical activity. Journal of Neural Engineering, 13(5), 056004. https://doi.org/10.1088/1741-2560/13/5/056004

Naselaris, T., Kay, K. N., Nishimoto, S., \& Gallant, J. L. (2011). Encoding and decoding in fMRI. NeuroImage, 56(2), 400-410. https://doi.org/10.1016/j.neuroimage.2010.07.073

Naselaris, T., Olman, C. A., Stansbury, D. E., Ugurbil, K., \& Gallant, J. L. (2015). A voxelwise encoding model for early visual areas decodes mental images of remembered scenes. NeuroImage, 105, 215-228. https://doi.org/10.1016/j.neuroimage.2014.10.018 
Nunez-Elizalde, A., Deniz, F., Gao, J., \& Gallant, J. (2018). Brain activity recorded during free viewing of naturalistic short films simultaneously reveals the brain representations of multiple feature spaces. Conference on Cognitive Computational Neuroscience, 5-8 September 2018, Philadelphia, Pennsylvania, PS-1B.11. https://doi.org/10.32470/ccn.2018.1072-0

Panzeri, S., Brunel, N., Logothetis, N. K., \& Kayser, C. (2010). Sensory neural codes using multiplexed temporal scales. Trends in Neurosciences, 33(3), 111-120. https://doi.org/10.1016/j.tins.2009.12.001

Panzeri, S., Harvey, C. D., Piasini, E., Latham, P. E., \& Fellin, T. (2017). Cracking the Neural Code for Sensory Perception by Combining Statistics, Intervention, and Behavior. Neuron, 93(3), 491-507. https://doi.org/10.1016/j.neuron.2016.12.036

Pasley, B. N., David, S. V, Mesgarani, N., Flinker, A., Shamma, S. A., Crone, N. E., Knight, R. T., \& Chang, E. F. (2012). Reconstructing speech from human auditory cortex. PLoS Biology, 10(1), e1001251. https://doi.org/10.1371/journal.pbio.1001251

Pereira, F., Lou, B., Pritchett, B., Ritter, S., Gershman, S. J., Kanwisher, N., Botvinick, M., \& Fedorenko, E. (2018). Toward a universal decoder of linguistic meaning from brain activation. Nature Communications, 9(1), 963. https://doi.org/10.1038/s41467018-03068-4

Poldrack, R. A., \& Yarkoni, T. (2016). From brain maps to cognitive ontologies: Informatics and the search for mental structure. Annual Review of Psychology, 67, 587-612. https://doi.org/10.1146/annurev-psych-122414-033729

Popov, V., Ostarek, M., \& Tenison, C. (2018). Practices and pitfalls in inferring neural representations. NeuroImage, 174, 340-351. https://doi.org/10.1016/j.neuroimage.2018.03.041

Pulvermüller, F., \& Fadiga, L. (2010). Active perception: sensorimotor circuits as a cortical basis for language. Nature Reviews Neuroscience, 11(5), 351-360. http://dx.doi.org/10.1038/nrn2811

Ritchie, J. B., Kaplan, D., \& Klein, C. (2017). Decoding The Brain: Neural Representation And The Limits Of Multivariate Pattern Analysis In Cognitive Neuroscience. The British Journal for the Philosophy of Science, 70(2), 581-607. https://doi.org/10.1101/127233

Roussel, P., Godais, G. Le, Bocquelet, F., Palma, M., Hongjie, J., Zhang, S., Giraud, A.-L., Mégevand, P., Miller, K., Gehrig, J., Kell, C., Kahane, P., Chabardés, S., \& Yvert, B. 

(2020). Observation and assessment of acoustic contamination of electrophysiological brain signals during speech production and sound perception. Journal of Neural Engineering, 17(5), 056028. https://doi.org/10.1088/1741$2552 / \mathrm{abb} 25 \mathrm{e}$

Rubin, A., Sheintuch, L., Brande-eilat, N., Pinchasof, O., Rechavi, Y., Geva, N., \& Ziv, Y. (2019). Revealing neural correlates of behavior without behavioral measurements. Nature Communications, 10(1), 4745.

Rutten, S., Santoro, R., Hervais-Adelman, A., Formisano, E., \& Golestani, N. (2019). Cortical encoding of speech enhances task-relevant acoustic information. Nature Human Behaviour, 3(9), 974-987.

Santoro, R., Moerel, M., De Martino, F., Goebel, R., Ugurbil, K., Yacoub, E., \& Formisano, E. (2014). Encoding of Natural Sounds at Multiple Spectral and Temporal Resolutions in the Human Auditory Cortex. PLoS Computational Biology, 10(1). https://doi.org/10.1371/journal.pcbi.1003412

Santoro, R., Moerel, M., De Martino, F., Valente, G., Ugurbil, K., Yacoub, E., \& Formisano, E. (2017). Reconstructing the spectrotemporal modulations of real-life sounds from fMRI response patterns. Proceedings of the National Academy of Sciences, 114(18), 4799-4804. https://doi.org/10.1073/pnas.1617622114

Saxe, A., Nelli, S., \& Summerfield, C. (2021). If deep learning is the answer, what is the question? Nature Reviews Neuroscience, 22(1), 55-67.

$$
\text { https://doi.org/10.1038/s41583-020-00395-8 }
$$

Sheng, J., Zheng, L., Lyu, B., Cen, Z., Qin, L., Tan, L. H., Huang, M.-X., Ding, N., \& Gao, J.-H. (2018). The Cortical Maps of Hierarchical Linguistic Structures during Speech Perception. Cerebral Cortex, 1-9. https://doi.org/10.1093/cercor/bhy191

Shibata, K., Lisi, G., Cortese, A., Watanabe, T., \& Sasaki, Y. (2019). Toward a comprehensive understanding of the neural mechanisms of decoded neurofeedback. NeuroImage, 188, 539-556. https://doi.org/10.1016/j.neuroimage.2018.12.022

Snoek, L., Miletic, S., \& Scholte, H. S. (2019). How to control for confounds in decoding analyses of neuroimaging data. NeuroImage, 184, 741-760. https://doi.org/10.1101/290684

Strijkers, K. (2016). Can hierarchical models display parallel cortical dynamics? A nonhierarchical alternative of brain language theory. Language, Cognition and 
Neuroscience, 31(4), 465-469. https://doi.org/10.1080/23273798.2015.1096403

Teige, C., Mollo, G., Millman, R., Savill, N., Smallwood, J., Cornelissen, P. L., \& Jefferies, E. (2018). Dynamic semantic cognition: Characterising coherent and controlled conceptual retrieval through time using magnetoencephalography and chronometric transcranial magnetic stimulation. Cortex, 103, 329-349. https://doi.org/10.1016/j.cortex.2018.03.024

van Gerven, M. A. J. (2017). A primer on encoding models in sensory neuroscience. Journal of Mathematical Psychology, 76, 172-183. https://doi.org/10.1016/j.jmp.2016.06.009

Van Wassenhove, V. (2009). Minding time in an amodal representational space. Philosophical Transactions of the Royal Society B: Biological Sciences, 364, 18151830. https://doi.org/10.1098/rstb.2009.0023

Varoquaux, G., \& Poldrack, R. A. (2019). Predictive models can overcome reductionism in cognitive neuroimaging. Current Opinion in Neurobiology, 55, 1-6. https://doi.org/10.1016/j.conb.2018.11.002

Varoquaux, G., \& Thirion, B. (2014). How machine learning is shaping cognitive neuroimaging. GigaScience, 3(1), 28. https://doi.org/10.1186/2047-217X-3-28

Venezia, J. H., Thurman, S. M., Richards, V. M., \& Hickok, G. (2019). Hierarchy of speechdriven spectrotemporal receptive fields in human auditory cortex. NeuroImage, 186, 647-666. https://doi.org/10.1016/j.neuroimage.2018.11.049

Zhang, Q., Hu, X., Hong, B., \& Zhang, B. (2019). A hierarchical sparse coding model predicts acoustic feature encoding in both auditory midbrain and cortex. PLoS Computational Biology, 15(2), 1-23. https://doi.org/10.1371/journal.pcbi.1006766

Zhang, Y., Han, K., Worth, R., \& Liu, Z. (2020). Connecting concepts in the brain by mapping cortical representations of semantic relations. Nature Communications, 11(1), 1-13. https://doi.org/10.1038/s41467-020-15804-w

Zylberberg, J. (2018). The role of untuned neurons in sensory information coding. BioRxiv, 1-23. 